\title{
An Optimized Deep Learning Based Technique for Grading and Extraction of Diabetic Retinopathy Severities
}

Qiu-Ming Zhang

School of Computer science, China University of Geosciences, Wuhan 430074, China

E-mail: qiumingzhang98@outlook.com

Jing Luo

Practice training center, Wuhan Vocational College of Software and Engineering, Wuhan 430205, China

E-mail: jingLuo12@hotmail.com

Korhan Cengiz

Department of Electrical - Electronics Engineering, Trakya University, 22030, Edirne, Turkey

E-mail: korhancengiz@trakya.edu.tr

Keywords: diabetic retinopathy, machine learning, deep learning, convolution neural network, severity grading

Received: May 21, 2021

The prognosis of Diabetic Retinopathy $(D R)$ requires regular eye examinations, as ophthalmologists depends on fundus segmentation to treat DR pathologies. Automated approaches for detection, segmentation and classification have developed as an imperative area of research for the effective diagnosis of DR for the treatment of serious eye conditions that prevent visual impairment. Diagnosis of various DR lesions, as well as different severities, helping the ophthalmologists to analyze variations in fundus images and take the necessary measures before the disease progresses. Deep learning techniques have evolved as a recent advent to combat the issues of conventional machine leaning based methods. An optimized deep learning framework is proposed in this article for grading and extraction of diabetic retinopathy severities. This involves various steps like background segmentation, feature set extraction, feature optimization using Cuckoo search and Convolutional Neural Network (CNN) severity grade classification. The method was validated on two standard datasets MESSIDOR and IDRiD. The proposed method yields an accuracy value of $97.55 \%$, cross entropy loss of 0.367 and time intricacy of 20 mins and 15 secs for MESSIDOR and $98.02 \%$ cross entropy loss of 0.345 and time intricacy of 22 mins and 21 secs for IDRiD dataset; respectively. The state-of-the-art comparison depicts that the proposed CNN based method provides a maximum accuracy improvement of $10.46 \%$ comparative to the existing methodology. The proposed framework yields better accuracy by procurement of the investigative outcomes acquired exhibits proficient DR determination.

Povzetek: Opisana je metoda ugotavljanja sladkorne bolezni iz slik očesnega ozadje na osnovi strojnega in globokega učenja.

\section{Introduction}

Prolonged diabetes leads to progressive microvascular complication, Diabetic Retinopathy (DR) which is the major source of serious vision impairments. DR cannot be recognized at the early stage but as the condition progresses, various lesions start appearing on retinal surface making early detection of disease, a crucial task. Various DR related lesions are depicted in Figure 1.

DR is classified into two different forms: NonProliferative DR (NPDR) and Proliferative DR (PDR), however, in this paper NPDR stages are considered as they include the early signs of retinopathy [1-3].

Different means are stated in the previous works for the identification of various DR lesion. A CLEAR-DR arrangement was anticipated by Devinder Kumar, et al. to enable the medical decision sustenance for DR [4]. The efficiency and usefulness of the anticipated system is established for the identification of DR which further improves the clinical practices. Ahmed ElTanboly, et al. developed a CAD based method for the grading of different DR stages [5]. A three-way Dr detection method was presented by Anam Tariq, et al. [6] for classification of different DR lesions. The system validation is done along with the assessment of various

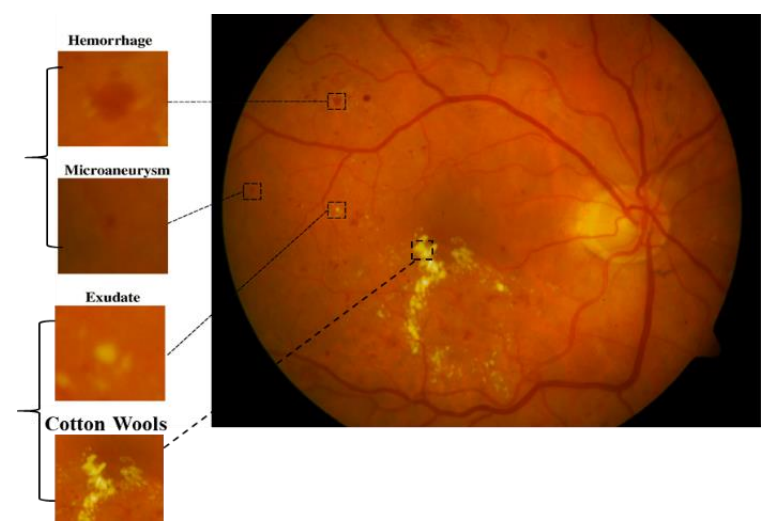

Figure 1: Different DR related Lesions. 
parameters to justify the viability of the anticipated technique. Sunrita Poddar, et al. [7] further presented a DR and maculopathy grading mechanisms involving various sequential steps. The classification is done based on the shape and degree of the lesions identified. Another grading mechanism was anticipated by Alan D. Fleming, et al. [8] for the detection of microaneurysms (MAs) and its further differentiation from the noise like spots. This method provides satisfactory outcomes for the identification of MAs.

Advances have been made in the field of medical imaging research and classification through the introduction of a new approach to bioimaging that relies on an ensemble of convolutional neural networks (CNNs). Li et al. [9] presented a DR classification approach using the pretrained networks tested on small datasets. Authors in [10] applied a CNN based transfer learning approach for DR recognition which yields much better sensitivity than the other conventional approaches. GoogleNet CNN platform was used by the authors in [11] in order to detect the symptoms of diabetic macular edema (DME) utilizing the benchmark MESSIDOR and the other is the E-Ophtha datasets. AlexNet based architecture was presented by the authors in [12] using the MESSIDOR dataset for diagnostic information extraction of DR severities. A zoon-in-Net based DR diagnostic platform was built by Wang, et al. [13] for retinopathy detection and severity grading. DR identification performance was improved by the authors in [14] using six different CNN architectures using the deep learning concept. A study was presented by the authors in [15] that compares the traditional DR classification methods to the deep learning-based approaches using various standard datasets. Authors in [16] developed a cross-disease review network for finding various specific characteristics of intrinsic relationship between diabetic macular edema (DME) and DR. Bhardwaj, et al. [17] presented a Quadrant based Inception-V3 network for DR grading using the two benchmark datasets (MESSIDOR and IDRiD). The methods proposed by then could be used for mass screening of DR and provide a second opinion to the ophthalmologists. Authors in [18] presented a significant DR grading method using IDRiD and MESSIDOR dataset considering five-fold validation of the CNN network. Various stages of pre-processing are used by the authors in order to transfer the architecture to various computational levels.

This literature survey of various techniques suggests that the deep learning-based strategies are being focused on in the present scenario in order to provide better results for various image recognition and classification approaches [19-21]. The DR classification-based approaches have also shifted their concern towards the deep learning as the previously used traditional methods were labor intensive, require professional information [22-24]. However, despite of various advantages of DNN methods, still it possesses the challenge for healthcare application and its practical implementation. In order to address such limitations, this article aims at evaluating the appropriateness of $\mathrm{CNN}$-enabled techniques for classifying DR into various DR severities and provide a significant contribution in medical imaging domain.

This article focuses on developing an optimized deep learning-based technique for grading and extraction of diabetic retinopathy severities which can automatically detect lesions and can assess its severity grade as mild, moderate or severe NPDR stage. The stages involved are background segmentation, feature set extraction, feature optimization and severity grade classification. Cuckoo search (CS) optimization algorithm is applied on the extracted feature attributes to obtain the Optimized Feature Set which can be used for CNN based DR severity categorization whose efficacy is observed experimentally by the computation of various evaluation parameters. The method was validated on MESSIDOR [25] and IDRiD [26] dataset. The proposed deep learning framework exhibits the feasible ability for proficient DR determination.

The remaining paper is systematized as follows: section 2 presents the methods and materials followed by the results and discussion of the proposed framework in section 3. Section 4 provides the concluding remarks of the article including the future considerations.

\section{Material and methods}

An optimized deep learning-based technique has been presented in this article for grading and extraction of diabetic retinopathy severities for providing a DR classification solution for retinal fundus images. The proposed framework which is divided into various successive steps signified in Figure 2.

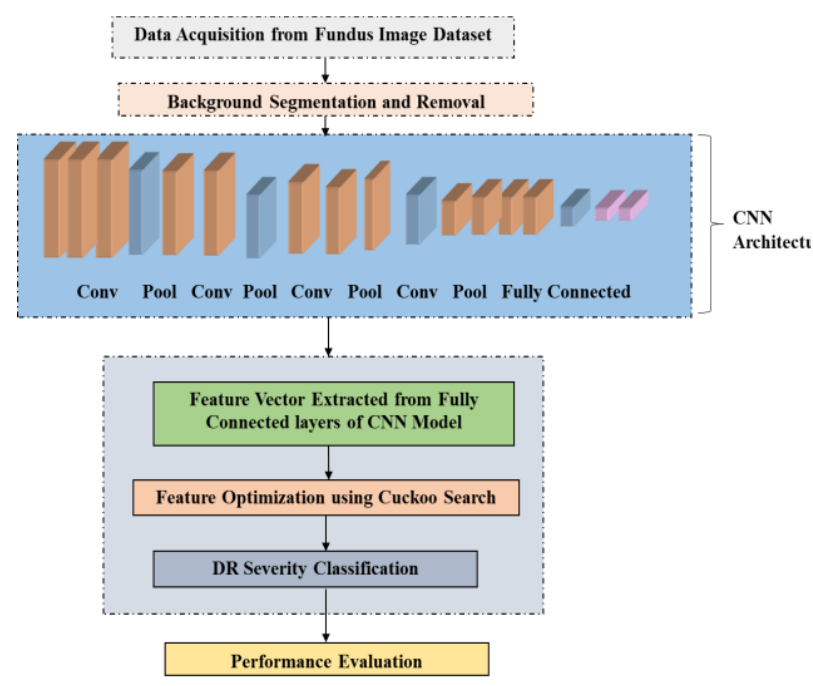

Figure 2: Proposed Framework for optimized deep learning based DR grading technique.

The undesired retinal components are masked using background segmentation and removal. These feature set is extracted from the final fully connected layers for the CNN architecture which are then are optimized using cuckoo search optimization algorithm and the optimized feature set is used for CNN based DR severity classification. The effectiveness of severity classification is experimentally observed using various evaluation 
parameters and block-wise explanation of the proposed methodology is detailed in the following section.

\subsection{Background segmentation and removal}

The raw fundus images are obtained from standard dataset MESSIDOR [25] and latest IDRiD dataset [26] to assess the DR abnormalities. The original fundus images involves several image related artefacts, thus, some of the image pre-processing techniques are required to diminish these flaws preceding to Optical Disc (OD) localization. The image pre-processing stages which are utilized for this experimental set-up consists of conversion of RGB intensities, histogram equalization followed by the segmentation of optical disc and blood vessel retinal components are removed primarily due to their less relevance for anomaly identification and classification. Some of these segmented component, if not removed at the primary stage may lead to misclassification due to its similarity with the pathological indicators. The training data for neural networks is very essential as lack of data and small dataset problem may lead to class imbalance. For removing this redundancy, various data augmentation methods are used in this study like horizontal and vertical flips, random rotation, random scaling in order to increase the training samples.

\subsection{Feature optimization}

The wide range of feature set is extracted from the final fully connected layers for the CNN architecture which are then are optimized using cuckoo search optimization algorithm. The Cuckoo Search (CS) optimization algorithm to provide Optimized Feature Set (OFS) to the classification stage. This approach is based on the reproduction process of cuckoo birds. Cuckoo birds lay their eggs in the nest of other host species so that their off-springs can be raised by these proxy parents. If these proxy parents discover about the alien eggs in their nests, these eggs are either thrown away or the entire nest is uninhibited. The algorithm of CS optimization is restricted to certain constraints.

Constraint 1: Individual cuckoo bird casually picks out a random nest and lays one egg in it.

Constraint 2: The nest comprising the high quality of eggs is further approved for the next generation, however, others are discarded.

Constraint 3: The probability of finding the alien egg by the host species is $p_{a} \in[0,1]$ and these alien eggs are either thrown away or the nest can be uninhibited.

Cuckoo search optimization algorithm is operative for solving global optimization problem and the major advantage of this algorithm is its simple implementation. It is less complex and efficient optimization approach as compared to other meta-heuristic approaches like particle swarm optimization and harmony search algorithms as it considers only single parameter [27].

The complete CS algorithm is given in Figure 3.

\subsection{CNN architecture for DR classification}

Objective function is defined as $f(x)$ where $x=$
$\left(x_{1}, x_{2}, \ldots \ldots, x_{i}\right)$
Create a initial population $x_{i}$ consisting of $n$ host nests
where $i=1,2, \ldots \ldots, n$
WHILE $(\mathrm{t}<$ Maximum Generation) or (stopping
criteria)
Get any $i_{t h}$ cuckoo arbitrarily by Levy distribution,
Assess its fitness function : $F_{i}$
Among the initial population of host nests, choose any
$j_{t h}$ nest randomly
Evaluate its fitness function : $F_{j}$
IF ( $\left.F_{i}>F_{j}\right)$
Replace $j$ with a new solution
END
A segment ( $\left.p_{a}\right)$ of inferior nests are uninhibited and the
new nests are constructed
Keep the best solutions/ nest
The best solutions are ranked in an order to find the
existing best solution
Permit the existing best solution to the next generation
END WHILE

Figure 3: Cuckoo Search Algorithm.

Convolutional deep neural networks are suited for imagebased retinopathy diagnostics. In this article, DR classification is accomplished using the CNN architecture. The basic CNN model depicted in Figure 4 consisting of an input layer followed by a pile of convolutional layers, several pooling-merging layers, fully connected layers, and an output layer to derive four different DR severities [28].

The basic CNN layers are explained below:

Convolution Layer: The convolution layer is designed to accept the input and then filter kernels are used followed

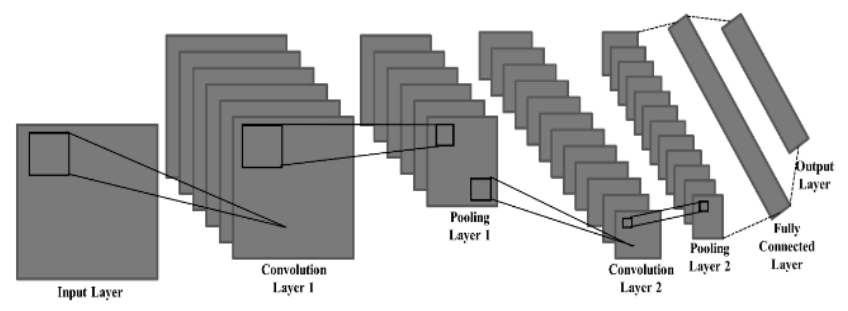

Figure 4: Basic Convolutional Neural Network architecture.

by the activation unit to obtain the output features. While considering $X_{\mathrm{i}}$ as the input image and $y_{i}$ as the output of convolutional operation. The function expressing the convolutional layer output is given by Eq. (1).

$$
y_{i}=\sum_{i=0}^{J} w_{i} * X_{i}+b
$$

where $w$ is weight vector, $i$ denoted the total nodes till the maximum number of node $J$ and $*$ denotes the convolutional operator.

Rectified Linear Unit (ReLU) is utilized to compute the maximum of the output obtained using the function $f(x)=\max (0, x)$.

Pooling-merging Layer: It is common to append the convolutional layer with the pooling layer after the 
convolution operation. This is done so as to obtain the down sampled output. The most commonly used pooling operator is max pooling that computes the local maximum of input features for intermediate dimensionality reduction.

Fully connected Layer: A series of fully connected layers are stacked subsequently after adjacent convolution and pool-merging layers. This particular stack of layer is responsible for facilitating the flattening of output into single vector for classification purpose. This layer uses the softmax activation function for computing the output and it is expressed by Eq. (2).

$$
\operatorname{Softmax}(X)=\frac{e^{m n}}{\sum_{n} e^{m n}}
$$

where $e^{m n}$ is exponential operator to obtain the output vector.

The CNN architecture used in this work for DR recognition and analysis consist of three subsequent convolutional and pooling layers. These layers consist of convolutional filters of size $5 \times 5$ and the pooling filters of size $2 \times 2$. The number of filters keeps in increasing from to increase the network depth. The parameter considerations of the network layers are tabulated in Table 1.

\begin{tabular}{cccc}
\hline Layer & Kernel Size & $\begin{array}{c}\text { Stride } \\
\text { Size }\end{array}$ & $\begin{array}{c}\text { Number of } \\
\text { Filters }\end{array}$ \\
\hline $\begin{array}{c}\text { Conv layer } \\
\mathbf{1}\end{array}$ & $5 \times 5 \times 1$ & 1 & 25 \\
$\begin{array}{c}\text { Pool layer } \\
\mathbf{1}\end{array}$ & $2 \times 2 \times 1$ & 2 & 25 \\
$\begin{array}{c}\text { Conv layer } \\
\mathbf{2}\end{array}$ & $5 \times 5 \times 25$ & 1 & 50 \\
$\begin{array}{c}\text { Pool layer } \\
\mathbf{2}\end{array}$ & $2 \times 2 \times 1$ & 2 & 50 \\
$\begin{array}{c}\text { Conv layer } \\
\mathbf{2}\end{array}$ & $5 \times 5 \times 50$ & 1 & 75 \\
$\begin{array}{c}\text { Pool layer } \\
\mathbf{2}\end{array}$ & $2 \times 2 \times 1$ & 2 & 75 \\
\hline
\end{tabular}

Table 1: Parameter considerations for the CNN architecture used in the proposed system.

\subsection{Performance evaluation parameters}

The performance of the presented CNN model for DR recognition and classification is measured for the metrics like accuracy, time intricacy, and cross entropy cost function are formulated in the following equations.

$i$. Accuracy: It defines the percentage of exactly corrected outcomes obtained out of the total number of events. The formula for accuracy is expressed by Eq. (3).

$$
A C C=\frac{T P+T N}{T P+T N+F P+F N}
$$

ii. Sensitivity: The percentage of accurately indicated chronic retinal diseased class cases which actually possess chronic DR symptoms are indicated by Sensitivity. It is expressed by Eq. (4).

$$
\text { Sensitivity }=\frac{T P}{T P+F N}
$$

iii. Specificity: Specificity provides the higher probability of truly indicated non-chronic DR class without giving the false positive outcomes. It is expressed by Eq. (5).

$$
\text { Specificity }=\frac{T N}{T N+F P}
$$

iv. Precision: This value indicated the correctly or precisely obtained accurate results from the total number of positive predictions. Precision is expressed by Eq. (6).

$$
\text { Precision }=\frac{T P}{T P+F P}
$$

v. Cross-Entropy: The cross-entropy loss is able to provide the correct classification capabilities of a neural network. The expression is given in Eq. (7).

$$
\begin{aligned}
& \text { Cross Entropy } \\
& =\frac{1}{\sum_{t=1}^{N} \text { Output }_{o, t} \log \left(p_{o}, t\right)}
\end{aligned}
$$

where $N=$ classes, true classification $=t$ for observation $o$, prediction probability $p$.

\section{Results and discussion}

The experimentation done for DR severity grading is done utilizing the optimized framework and the network performance is accessed using several evaluation parameters and the cost analysis is done in terms of cross entropy as well as time intricacy. The images are taken from both the datasets: MESSIDOR and IDRiD which are initially background segmented followed by the optimized feature set reduction using the Cuckoo search methodology. The outcomes of background segmentation and removal are depicted in Figure 5.

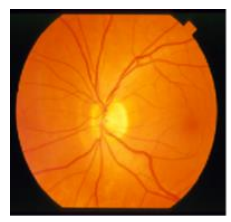

(a)

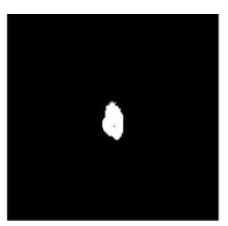

(b)

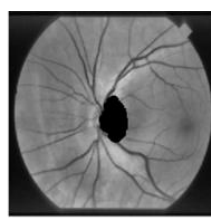

(c)
Figure 5: (a) Original image, (b) OD segmented part (c) Retinal image after segmentation.

The proposed framework the CNN classification network and the performance assessment in terms of various performance parameters are shown in Figure 6.

The comparison done in Figure 6 indicates that the favorable outcomes are achieved with the performance parameters as; $97.55 \%$ accuracy, $98.04 \%$ sensitivity, $97.06 \%$ specificity and $97.09 \%$ precision value for MESSIDOR dataset and $98.02 \%$ accuracy, $98.04 \%$ sensitivity, $98.00 \%$ specificity and $98 \%$ precision value for IDRiD dataset.

The analysis of cost effectiveness is done in regards of cross entropy and time intricacy. For a reliable performance of a CNN network, these values should be 
minimum. The graphical presentation of these parameters in done in Figure 7.

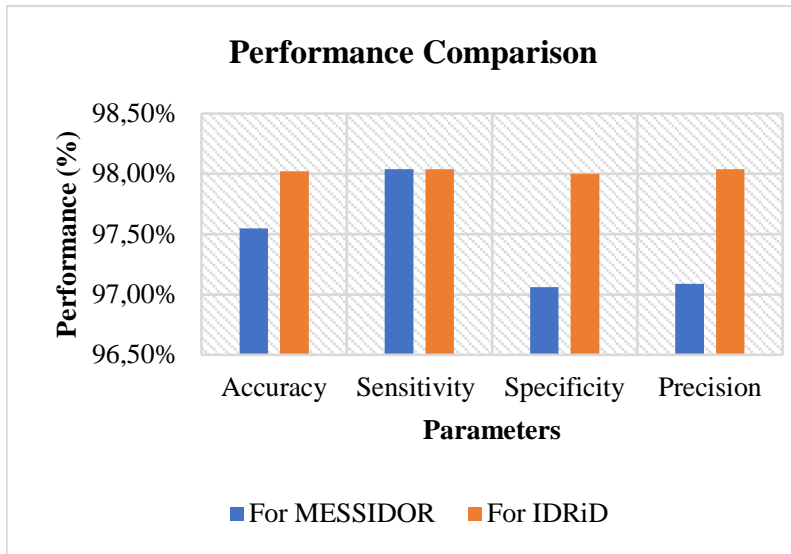

Figure 6: Performance parameters obtained for proposed framework.

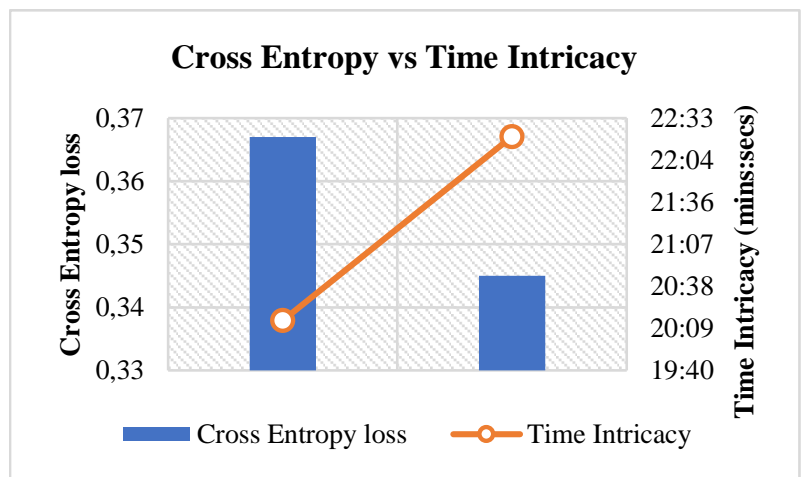

Figure 7: Cost analysis outcomes for cross entropy and time intricacy.

The cross-entropy loss of 0.367 is obtained for time intricacy of 20 minutes and 15 seconds for MESSIDOR dataset and 0.345 cross entropy loss with time intricacy of 22 minutes and 21 seconds for IDRiD dataset. As the cross entropy reduces for the proposed method, time intricacy increases, thereby, maintaining a tradeoff.

\subsection{Comparison with other methods}

The comparative analysis is done two way in this article. Firstly, the pretrained CNN models are compared with the proposed model in order to validate its flexibility and performance. The various pretrained CNN models used are AlexNet [29], GoogleNet [30], ResNet [31] and VggNet [32, 33]. The comparison is drawn in terms of time intricacy and accuracy which are shown in Figure 8.

This comparative analysis reveals that the proposed method withstands among all the pretrained model providing the maximum performance for DR severity grading in terms of accuracy. However, the time intricacy comparison reveals that proposed framework maintains a trade-off in this case and is a bit complex comparative to the other CNN pretrained architectures.

In this article, a comparative analysis with the stateof-the-art methods indicated in literature is also done in terms of percentage accuracy improvement. The comparison is drawn in Figure 9.

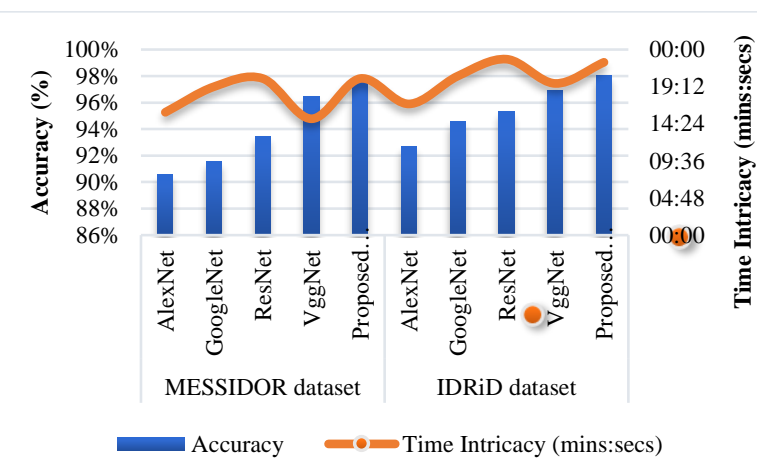

Figure 8: Comparison with other $\mathrm{CNN}$ pretrained models.

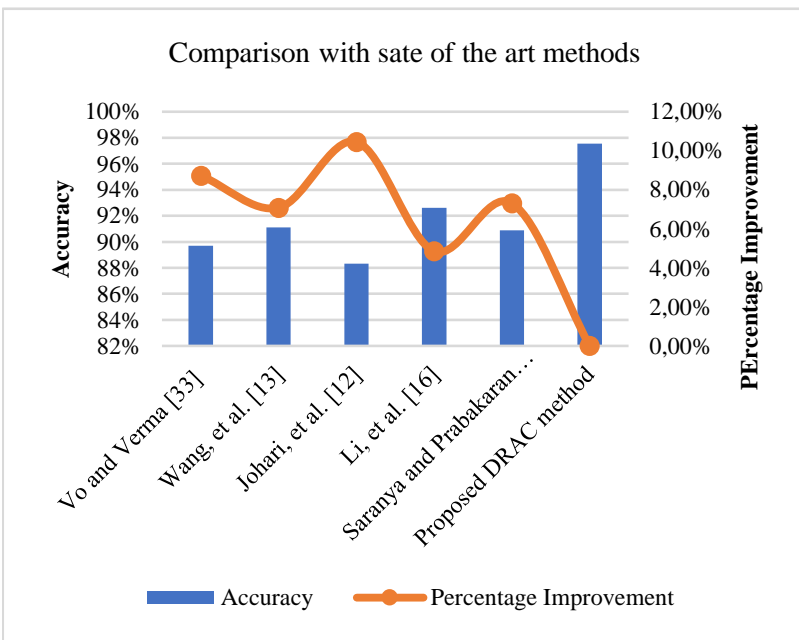

Figure 9: Comparison of existing state-of-the-art methods with the proposed framework.

The competency of the proposed optimized approach for DR severity grading is assessed in terms of percentage accuracy improvement. It provides reliable solution with maximum percentage improvement of $10.46 \%$ from the state-of-the-art literary work.

\section{Conclusions}

This article proposes an optimized deep learning-based technique for grading and extraction of diabetic retinopathy severities. The qualitative and quantitative analysis is based on various performance parameters as well as cost analysis. The two benchmark datasets evaluated for the study are MESSIDOR and IDRiD. The background segmentation, feature set extraction, feature optimization and $\mathrm{CNN}$ based severity grade classification stages are involved in this work for improving the network performance. The proposed method yields an accuracy value of $97.55 \%$ for MESSIDOR and $98.02 \%$ for IDRiD dataset maintaining a cross entropy loss of 0.367 and 0.345 respectively. The time intricacy of 20 mins and 15 secs is observed for MESSIDOR whereas 22 mins and 21 secs are seen for IDRiD dataset which maintains a tradeoff with the network performance. The state-of-the-art comparison reveals the feasibility of the proposed classification method which provides a maximum accuracy improvement of $10.46 \%$ with the 
currently prevailing methodology. The proposed technique can be used in the future for providing a reliable solution enabling the deep learning concept for providing the real time DR severity grading services to the ophthalmologists.

\section{References}

[1] Akram, M. U., Khalid, S., Tariq, A., Khan, S. A., \& Azam, F. (2014). Detection and classification of retinal lesions for grading of diabetic retinopathy. Computers in biology and medicine, 45, 161-171. https://doi.org/10.1016/j.compbiomed.2013.11.014.

[2] Fleming, A. D., Philip, S., Goatman, K. A., Olson, J. A., \& Sharp, P. F. (2006). Automated microaneurysm detection using local contrast normalization and local vessel detection. IEEE transactions on medical imaging, 25(9), 1223-1232. https://doi.org/10.1109/tmi.2006.879953.

[3] ElTanboly, A., Ghazal, M., Khalil, A., Shalaby, A., Mahmoud, A., Switala, A., ... \& El-Baz, A. (2018, April). An integrated framework for automatic clinical assessment of diabetic retinopathy grade using spectral domain OCT images. In 2018 IEEE 15th International Symposium on Biomedical Imaging (ISBI 2018), pp. 1431-1435. IEEE. https://doi.org/10.1109/ISBI.2018.8363841.

[4] Kumar, D., Taylor, G. W., \& Wong, A. (2019). Discovery radiomics with CLEAR-DR: interpretable computer aided diagnosis of diabetic retinopathy. IEEE Access, 7, 25891-25896. https://doi.org/10.1109/ACCESS.2019.2893635.

[5] ElTanboly, A., Ismail, M., Shalaby, A., Switala, A., El-Baz, A., Schaal, S., ... \& El-Azab, M. (2017). A computer-aided diagnostic system for detecting diabetic retinopathy in optical coherence tomography images. Medical physics, 44(3), 914923. https://doi.org/10.1002/mp.12071.

[6] Tariq, A., Akram, M. U., Shaukat, A., \& Khan, S. A. (2013). Automated detection and grading of diabetic maculopathy in digital retinal images. Journal of digital imaging, 26(4), 803-812. https://doi.org/10.1007/s10278-012-9549-4.

[7] Poddar, S., Jha, B. K., \& Chakraborty, C. (2011, November). Quantitative clinical marker extraction from colour fundus images for non-proliferative diabetic retinopathy grading. In 2011 International Conference on Image Information Processing, pp. 16. IEEE. https://doi.org/10.1109/ICIIP.2011.6108956.

[8] Fleming, A. D., Goatman, K. A., Philip, S., Williams, G. J., Prescott, G. J., Scotland, G. S., ... \& Olson, J. A. (2010). The role of haemorrhage and exudate detection in automated grading of diabetic retinopathy. British Journal of Ophthalmology, 94(6), 706-711. https://doi.org/10.1136/bjo.2008.149807.

[9] Li, X., Pang, T., Xiong, B., Liu, W., Liang, P., \& Wang, T. (2017, October). Convolutional neural networks based transfer learning for diabetic retinopathy fundus image classification. In 2017 10th international congress on image and signal processing, biomedical engineering and informatics (CISP-BMEI) (pp. 1-11). IEEE. https://doi.org/10.1109/CISP-BMEI.2017.8301998.

[10] García-Zurdo, Rubén. "Creation of facial composites from user selections using image gradient." Informatica 44, no. 1 (2020).

https://doi.org/10.31449/inf.v44i1.2340

[11] Perdomo, O., Otalora, S., Rodríguez, F., Arevalo, J., \& González, F. A. (2016). A novel machine learning model based on exudate localization to detect diabetic macular edema. https://doi.org/110.17077/omia.1057.

[12] Johari, M. H., Hassan, H. A., Yassin, A. I. M., Tahir, N. M., Zabidi, A., Rizman, Z. I., ... \& Wahab, N. A. (2018). Early detection of diabetic retinopathy by using deep learning neural network. International Journal of Engineering and Technology (UAE), 7(4), 198-201. https://doi.org/10.14419/ijet.v7i4.11.20804.

[13] Shijina, V., Adithya Unni, and Sunil Jacob John. "Similarity measure of multiple sets and its application to pattern recognition." Informatica 44, no. 3 (2020).

DOI: https://doi.org/10.31449/inf.v44i3.2872

[14] Chen, Y. W., Wu, T. Y., Wong, W. H., \& Lee, C. Y. (2018, April). Diabetic retinopathy detection based on deep convolutional neural networks. In 2018 IEEE international conference on acoustics, speech and signal processing (ICASSP), pp. 1030-1034. IEEE. https://doi.org/10.1109/ICASSP.2018.8461427.

[15] Gonçalves, J., Conceiçao, T., \& Soares, F. (2019). Inter-observer Reliability in Computer-aided Diagnosis of Diabetic Retinopathy. In HEALTHINF (pp. 481-491). https://doi.org/10.5220/0007580904810491.

[16] Li, X., Hu, X., Yu, L., Zhu, L., Fu, C. W., \& Heng, P. A. (2019). CANet: cross-disease attention network for joint diabetic retinopathy and diabetic macular edema grading. IEEE transactions on medical imaging, 39(5), 1483-1493.

https://doi.org/10.1109/TMI.2019.2951844.

[17] Bhardwaj, C., Jain, S., \& Sood, M. (2021). Hierarchical severity grade classification of nonproliferative diabetic retinopathy. Journal of Ambient Intelligence and Humanized Computing, 12(2), 2649-2670. https://doi.org/10.1007/s12652-020-02426-9.

[18] Saranya, P., \& Prabakaran, S. (2020). Automatic detection of non-proliferative diabetic retinopathy in retinal fundus images using convolution neural network. Journal of Ambient Intelligence and Humanized Computing, 1-10. https://doi.org/10.1007/s12652-020-02518-6.

[19] Dhiman, G., Oliva, D., Kaur, A., Singh, K. K., Vimal, S., Sharma, A., \& Cengiz, K. (2021). BEPO: A novel binary emperor penguin optimizer for automatic feature selection. Knowledge-Based Systems, 211, 106560. https://doi.org/10.1016/j.knosys.2020.106560. 
[20]Dhiman, G., Singh, K. K., Soni, M., Nagar, A., Dehghani, M., Slowik, A., ... \& Cengiz, K. (2021). MOSOA: a new multi-objective seagull optimization algorithm. Expert Systems with Applications, 167, 114150. https://doi.org/10.1016/j.eswa.2020.114150.

[21] Bhardwaj, C., Jain, S., \& Sood, M. (2021). Hierarchical severity grade classification of nonproliferative diabetic retinopathy. Journal of Ambient Intelligence and Humanized Computing, 12(2), 2649-2670. https://doi.org/10.1007/s12652-020-02426-9.

[22] Bhardwaj, C., Jain, S., \& Sood, M. (2020). Diabetic retinopathy lesion discriminative diagnostic system for retinal fundus images. Advanced Biomedical Engineering, 9, 71-82. https://doi.org/10.14326/abe.9.71.

[23] Rathee, G., Sharma, A., Kumar, R., Ahmad, F., \& Iqbal, R. (2020). A trust management scheme to secure mobile information centric networks. Computer Communications, 151, 66-75. https://doi.org/10.1016/j.comcom.2019.12.024.

[24] Yuvaraj, N., Srihari, K., Dhiman, G., Somasundaram, K., Sharma, A., Rajeskannan, S., ... \& Masud, M. (2021). Nature-Inspired-Based Approach for Automated Cyberbullying Classification on Multimedia Social Networking. Mathematical Problems in Engineering, 2021. https://doi.org/10.1155/2021/6644652.

[25] Sharma, A. and Kumar, N., 2021. Third Eye: An Intelligent and Secure Route Planning Scheme for Critical Services Provisions in Internet of Vehicles Environment. IEEE Systems Journal. DOI: https://doi.org/10.1109/JSYST.2021.3052072

[26] Porwal, P., Pachade, S., Kamble, R., Kokare, M., Deshmukh, G., Sahasrabuddhe, V., \& Meriaudeau, F. (2018). Indian diabetic retinopathy image dataset (IDRiD): a database for diabetic retinopathy screening research. Data, 3(3), 25. https://doi.org/10.3390/data3030025.

[27] Mareli, M., \& Twala, B. (2018). An adaptive Cuckoo search algorithm for optimisation. Applied computing and informatics, 14(2), 107-115. https://doi.org/ 10.1016/j.aci.2017.09.001.

[28] Gao, Z., Li, J., Guo, J., Chen, Y., Yi, Z., \& Zhong, J. (2018). Diagnosis of diabetic retinopathy using deep neural networks. IEEE Access, 7, 3360-3370. https://doi.org/10.1109/ACCESS.2018.2888639.

[29]Li, G., Liu, F., Sharma, A., Khalaf, O.I., Alotaibi, Y., Alsufyani, A. and Alghamdi, S., 2021. Research on the Natural Language Recognition Method Based on Cluster Analysis Using Neural Network. Mathematical Problems in Engineering, 2021.

DOI: https://doi.org/10.1155/2021/9982305

[30] Keshavarz-Ghorabaee, M., Amiri, M., Zavadskas, E. K., Turskis, Z., \& Antucheviciene, J. (2018). Simultaneous evaluation of criteria and alternatives (SECA) for multi-criteria decision-making. Informatica, 29(2), 265-280, DOI: https://doi.org/10.15388/Informatica.2018.167
[31]He, K., Zhang, X., Ren, S., \& Sun, J. (2016). Deep residual learning for image recognition. In Proceedings of the IEEE conference on computer vision and pattern recognition (pp. 770-778). https://doi.org/10.1109/CVPR.2016.90.

[32] Sayago-Heredia, J., Pérez-Castillo, R., \& Piattini, M. (2021). A Systematic Mapping Study on Analysis of Code Repositories. Informatica, 1-42, DOI: https://doi.org/10.15388/21-INFOR454

[33]Liu, L., Wang, L., Xu, D., Zhang, H., Sharma, A., Tiwari, S., ... \& Shah, M. A. (2021). CT Image Segmentation Method of Liver Tumor Based on Artificial Intelligence Enabled Medical Imaging. Mathematical Problems in Engineering, 2021. https://doi.org/10.1155/2021/9919507 
